\title{
Multitude of dimple shapes can produce singular jets during the collapse of immiscible drop-impact craters
}

\author{
Zi Qiang Yang ${ }^{1}$, Yuan Si Tian ${ }^{1}$ and S. T. Thoroddsen ${ }^{1} \uparrow$ \\ ${ }^{1}$ Division of Physical Science and Engineering, King Abdullah University of Science and Technology \\ (KAUST), Thuwal 23955-6900, Saudi Arabia
}

(Received 9 March 2020; revised 1 August 2020; accepted 13 August 2020)

We study singular jets from the collapse of drop-impact craters, when the drop and pool are of different immiscible liquids. The fastest jets emerge from a dimple at the bottom of the rebounding crater, when no bubble is pinched off. The parameter space is considerably more complex than for identical liquids, revealing intricate compound-dimple shapes. In contrast to the universal capillary-inertial drop pinch-off regime, where the neck radius scales as $R \sim t^{2 / 3}$, for a purely inertial air dimple the collapse has $R \sim t^{1 / 2}$. The bottom dimple dynamics is not self-similar but possesses memory effects, being sensitive to initial and boundary conditions. Sequence of capillary waves can therefore mould the air dimple into different collapse shapes, such as bamboo-like and telescopic forms. The finest jets are only $12 \mu \mathrm{m}$ in diameter and the normalized jetting speeds are up to one order of magnitude larger than for jets from bursting bubbles. We study the cross-over between the two power laws approaching the singularity. The singular jets show the earliest cross-over into the inertial regime. The fastest jets can pinch off a toroidal micro-bubble from the cusp at the base of the jet.

Key words: breakup/coalescence

\section{Introduction}

Singularities occur in many branches of physics from the gravitational collapse of a black hole (Chandrasekhar 1992; Choptuik 1993) to the pinch-off of a drop from a faucet (Brenner et al. 1997; Eggers 1997; Eggers \& Fontelos 2015). The reduced length and time scales near the singularity expose the important force balance governing the dynamics. The pinch-off of a drop from a nozzle was shown by Day, Hinch \& Lister (1998) to have a self-similar conical shape with capillary-inertial scaling of the necking radius versus time, $R \sim\left(\sigma t^{2} / \rho\right)^{1 / 3}$, where $\sigma$ is surface tension and $\rho$ the liquid density. In contrast 
the pinch-off of a bubble follows a purely inertial process with $R \sim\left(t_{c}-t\right)^{1 / 2}$ (Burton, Waldrep \& Taborek 2005; Eggers et al. 2007; Thoroddsen, Etoh \& Takehara 2007a). This modest difference in exponent values hides a profound difference in the dynamical nature of the pinch-off. For the purely inertial scaling, the surface tension becomes irrelevant near the final pinch-off and there is a strong dependence on the initial or boundary conditions. This memory of the boundaries has been best demonstrated for the pinch-off of a bubble from an elliptic nozzle (Schmidt et al. 2009; Lai 2012). Air cavities formed by the impact of circular plates with periodic edges produce kindred non-axisymmetric shapes, which can even split into multiple necks (Enriquez et al. 2012).

For the collapse of impact craters, Thoroddsen et al. (2018) have recently shown that the finest singular jets emerge from a dimple collapse with close to inertial scaling.

Fine jets can emerge from a free surface in numerous configurations, such as: the oscillation of a free-falling drop pinched off from a nozzle (Thoroddsen, Etoh \& Takehara 2007b); from a bursting bubble at a pool surface (Duchemin et al. 2002; Walls, Henaux \& Bird 2015; Deike et al. 2018; Lai, Eggers \& Deike 2018); from shock-accelerated curved interfaces (Antkowiak et al. 2007; Thoroddsen et al. 2009; Tagawa et al. 2012); following a drop impact on a superhydrophobic surface (Bartolo, Josserand \& Bonn 2006); during cylindrical collapse of sphere-impact craters (Gekle et al. 2009) and from critical Faraday waves in vertically oscillated liquid layers (Longuet-Higgins 1983; Zeff et al. 2000; Das \& Hopfinger 2008).

Herein, we study jets forming by the collapse of hemispheric drop-impact craters. While numerous studies have looked at the crater collapse when the drop and pool are of the same liquid (Pumphrey \& Elmore 1990; Prosperetti \& Oguz 1993; Liow 2001; Thoroddsen et al. 2018), few have studied a drop impacting a pool of a different immiscible liquid. Earlier work has mostly focused on drop deformation (Fujimatsu et al. 2003), fragmentation into smaller droplets (Lhuissier et al. 2013), a novel type of double entrainment (Jain et al. 2019) or breakup of oil spills by rain (Murphy et al. 2015). We will show that the landscape for singular jetting becomes much more complicated in the immiscible case.

\section{Experimental set-up}

The overall set-up is sketched in figure $1(c)$ and is similar to that used in previous studies on this topic by Thoroddsen et al. (2018). The drop pinches off from a flat stainless steel nozzle and falls onto a pool surface contained in a square glass container $(5 \mathrm{~cm} \times 5 \mathrm{~cm} \times$ $5 \mathrm{~cm}$ ). A syringe pump is used to feed the drop at a slow flow rate of $10 \mu 1 \mathrm{~min}^{-1}$. The drop diameters are less than $2 \mathrm{~mm}$, so capillary waves are not reflected from the tank wall to influence the impact dynamics. Our well-controlled experiments exhibited extreme sensitivity to boundary conditions, as has been reported in Thoroddsen et al. (2018) and Michon, Josserand \& Séon (2017), who call the jetting 'barely reproducible'.

Herein, we use two immiscible liquids (table 1). The pool is deionized (DI) water (Milli-Q), while the drop consists of PP1 (Perfluorohexane, $\mathrm{C}_{6} \mathrm{~F}_{14}$, from F2 Chemicals Ltd). The PP1 is 1.71 times heavier than water and has a very low surface tension $\sigma_{d}=11.9 \mathrm{mN} \mathrm{m}^{-1}$, as measured in house with a ring tensiometer (K100MK2/SF/C, Kruss $\mathrm{GmbH}$, Hamburg). The interfacial tension between water and PP1 is $48 \mathrm{mN} \mathrm{m}^{-1}$. The large difference in the refractive index makes the interface between the drop and pool clearly visible.

We use a range of PP1 drop sizes $D=0.60,0.72,0.85,0.95,1.2,1.5$ and 2.0 $\mathrm{mm}$. For reference, the capillary length for PP1 is $\sqrt{\sigma /(\rho g)}=0.84 \mathrm{~mm}$. By varying the drop release height we produce impact velocities $U$ between 0.1 and $3.9 \mathrm{~m} \mathrm{~s}^{-1}$. 
(a)
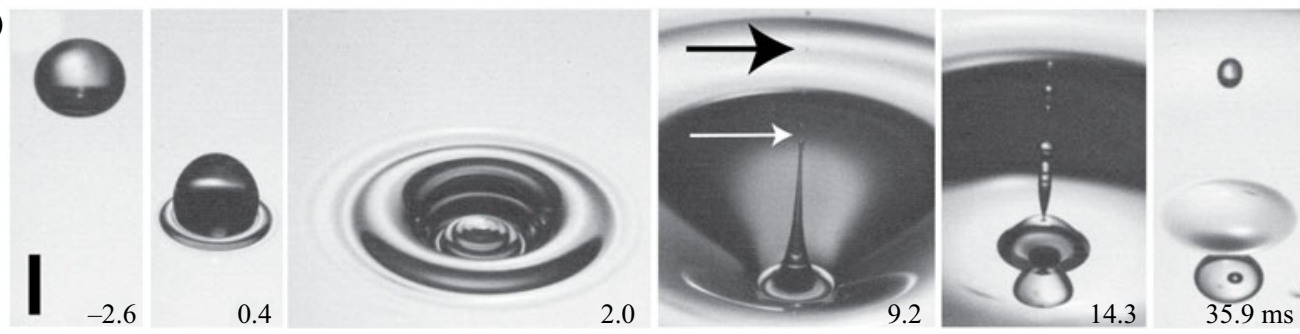

(b)
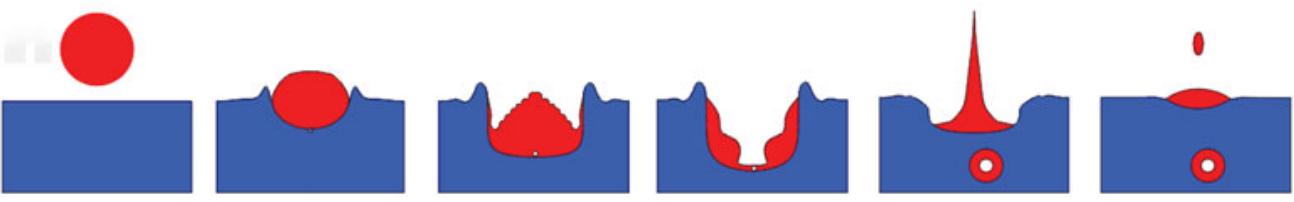

(c)

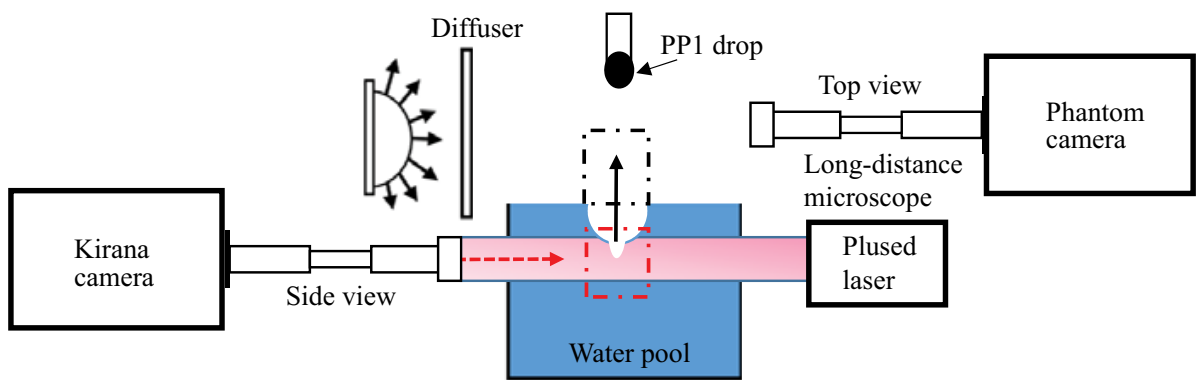

FIGURE 1. (a) Movie frames showing an angled top view of typical impact crater collapse and jetting, for $D=1.56 \mathrm{~mm}, U=1.04 \mathrm{~m} \mathrm{~s}^{-1}$, corresponding to $R e=3418$ and $W e=242$. The scale bar is $1 \mathrm{~mm}$ long. See also supplementary movie 1 available at https://doi.org/10.1017/ jfm.2020.694. (b) Sketch of the drop impact, crater evolution, jetting and bubble entrapment. (c) Experimental set-up, with two high-speed video cameras viewing from perpendicular directions, one for the dimple dynamics inside the pool and a second to view the jet droplets as they emerge out of the crater, as indicated by the arrow.

$\begin{array}{lccccc}\text { Liquid } & \begin{array}{c}\text { Density } \\ \rho\left(\mathrm{g} \mathrm{cm}^{-3}\right)\end{array} & \begin{array}{c}\text { Viscosity } \\ \mu(\mathrm{mPa})\end{array} & \begin{array}{c}\text { Surface tension } \\ \sigma\left(\mathrm{mN} \mathrm{m}^{-1}\right)\end{array} & \begin{array}{c}\text { Capillary length } \\ L_{c}(\mathrm{~mm})\end{array} & \begin{array}{c}\text { Refractive index } \\ n\end{array} \\ \text { PP1 } & 1.71 & 0.81 & 11.9 & 0.84 & 1.25 \\ \text { DI water } & 0.996 & 1.004 & 72.1 & 2.72 & 1.33\end{array}$

TABLE 1. Liquid properties of the PP1 drop and water pool.

The corresponding range of Reynolds, Weber and Froude numbers, based on drop liquid properties are

$$
\begin{gathered}
R e=\frac{\rho_{d} D U}{\mu_{d}}=374-10200, \\
W e=\frac{\rho_{d} D U^{2}}{\sigma_{d}}=10-2000, \\
F r=\frac{U^{2}}{g D}=10-1500,
\end{gathered}
$$

where $g$ is gravity, $\rho_{d}$ and $\mu_{d}$ drop density and dynamic viscosity. 
Two high-speed cameras simultaneously observe the crater collapse and jetting. The top camera (Phantom V2511) focuses on the jet rising above the liquid pool surface, while the other one studies the crater collapse below the surface. The bottom Kirana camera can reach $5 \mathrm{Mfps}$ at $\sim 1 \mu \mathrm{mpx}^{-1}$ resolution when using a long-distance microscope (Leica Z16 APO). Back lighting is produced by $350 \mathrm{~W}$ Sumita metal-halide lamp shone onto a diffuser, or by pulsed laser diodes (SI-LUX640, Specialized Imaging) at the highest frame rates.

\section{Results}

The impact forms a hemispheric crater into the pool surface, with the drop liquid stretched out into a thin continuous layer coating its surface. The subsequent rebound can form a bottom dimple whose collapse pinches off a bubble, or can produce singular jets (Rein 1996; Liow 2001; Michon et al. 2017; Thoroddsen et al. 2018). This is shown by the sequence of movie frames and sketches in figure $1(a, b)$ and the sequence in figure 2, showing the jet emergence and its break-up above the pool surface. The free surface of the bottom dimple therefore remains between the air and the PP1 drop liquid and has low surface tension. Figure 3 shows the parameter regime where a dimple forms at the bottom of the crater during its collapse. The boundary of this regime is marked by solid black lines. This occurs at a much larger We (based on drop properties), than for the classical regime (marked by dashed blue lines) where the bottom dimple entraps a bubble for identical liquids in both drop and pool (Pumphrey \& Elmore 1990; Prosperetti \& Oguz 1993). The phase diagram in figure $3(b)$ classifies the impact outcomes within the dimple-formation region, which is bounded between the two solid black curves. There is no air-dimple pinch-off above the upper bound and below the lower bound. Note that, because the surface tension and density of the drop liquid are constant in the experiments, by increasing the impact velocity, for a fixed drop diameter, one traces out a straight line from the origin, in the $\mathrm{We}-\mathrm{Fr}$ diagram. The intermediate region, between the curves is classified into different dimple shapes. Not only do we see pinch-off of a single bubble, but other more complex shapes are produced, owing to capillary waves travelling down the dimple. There are prominent bamboo-shaped dimples which appear in a subregion marked by the cyan dashed lines. Adjacent to this region we see the formation of telescopic dimples, marked by black stars, where no bubble is pinched off but the narrowest and fastest jets are observed. Furthermore, we find singular jets occurring at numerous locations within the dimple regime. This is in sharp contrast with crater collapse for cases when the drop and pool are of the same liquid (Rein 1996; Thoroddsen et al. 2018), where jets form only at the boundaries of the dimple regime. For example, in figure $3(c)$ we show an enlargement of the region within the red dashed rectangle in figure $3(b)$, where the large symbols show three different impact conditions with singular jets at different $W e$, as shown later in figure 9.

Keep in mind that the region close to the lower bound of the dimple-formation regime in figure $3(b)$, corresponds to the smallest drops, $D \simeq 0.7-0.9 \mathrm{~mm}$, which are close to the size of the capillary length $0.82 \mathrm{~mm}$. The oscillations of the free-falling drop, away from spherical shape, will therefore be minimal and unlikely to affect the crater dynamics significantly, as can occur for larger drops. We refer to Thoraval, Li \& Thoroddsen (2016), where the shape at first contact determines whether a large bubble is entrapped or not.

For impacts above this bubble-entrapment regime, we see the bottom of the drop form into a cylindrical liquid dimple which pinches off, as shown in figure 4(a); see also Lhuissier et al. (2013). Here the bottom of the air cavity retracts upwards 


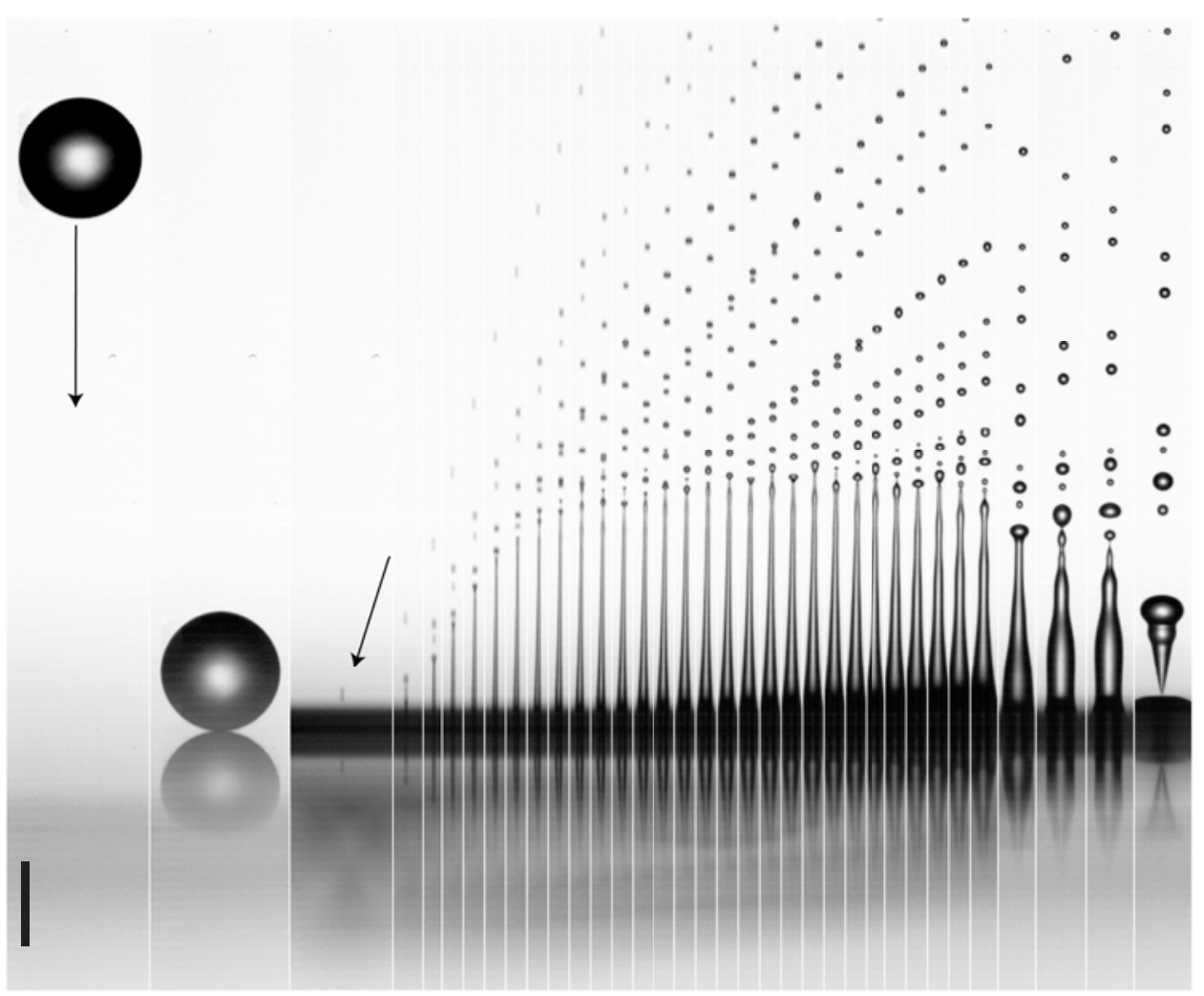

FIGURE 2. Overall shape and break-up of the fine jet emerging out of the impact crater for a PP1 droplet of $D=0.71 \mathrm{~mm}$, impacting on the water pool at velocity $U=1.18 \mathrm{~m} \mathrm{~s}^{-1}$, giving $R e=1779, F r=201, W e=143$. This corresponds to the first singular jet for this drop size, emerging at a jet velocity of $v_{j e t}=7.59 \mathrm{~m} \mathrm{~s}^{-1}$. The total number of shed droplets is here 21 . The movie is taken at $70 \mathrm{kfps}$ and the second panel shows the drop hitting the free surface. The first tip droplet is $21 \mu \mathrm{m}$ wide and emerges in the third panel (arrow) at $2.2 \mathrm{~ms}$ after the impact. Subsequent frames are separated by $57 \mu \mathrm{s}$, with the last four frames at $t=4.14$, $4.48,4.57$ and $4.66 \mathrm{~ms}$ after impact. The scale bar is $500 \mu \mathrm{m}$ long. See also supplementary movie 2.

without bubble pinch-off. However, the PP1 cylinder pinches off a satellite droplet inside the water pool through Rayleigh-Plateau instability. The reversed air cavity shown in the third frame of figure $4(a)$ is reminiscent of the secondary-bubble-entrapment dimple of Liow \& Cole (2007), even though there is no bubble pinch-off in our case.

In figure 4(b), the bottom surface of the PP1 column shows a reverse-curvature PP1-water interface. The dynamics engulfs some of the water to form a water-PP1 compound droplet, which is indicated by the black arrow in figure $4(b)$. A related process is found in the study of Terwagne et al. (2009), where they bounce a water drop coated with a thin silicon oil layer on a high-viscosity pool surface. The deformation of the drop entraps some of the surface oil film into the water drop, thereby forming a double emulsion. See also the engulfment of a pool droplet inside a contracting air disc, which is entrapped under an impacting drop (Jian et al. 2020). 
(a)
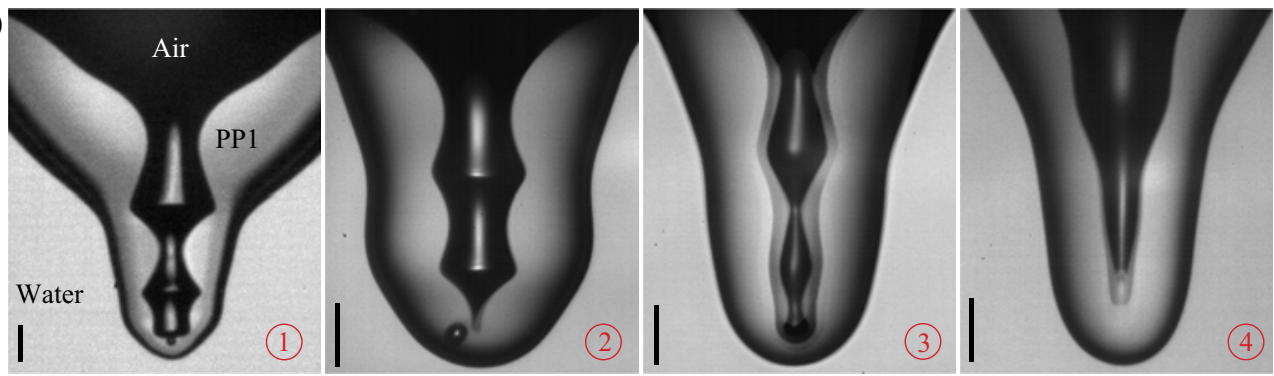

(b)

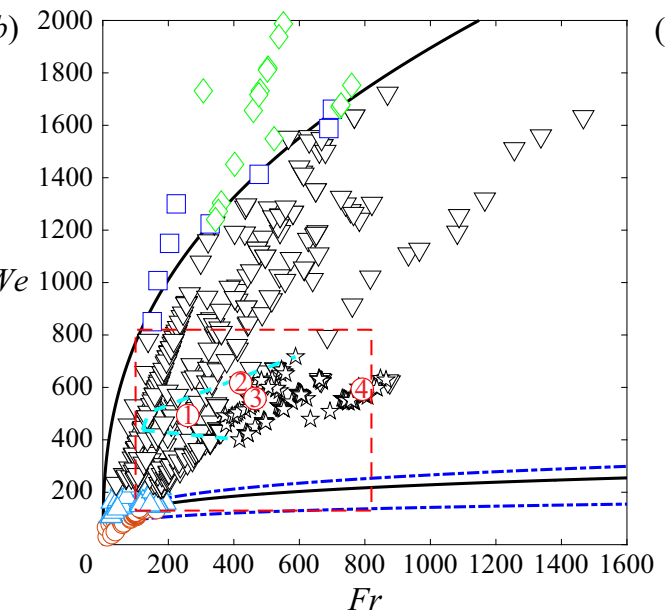

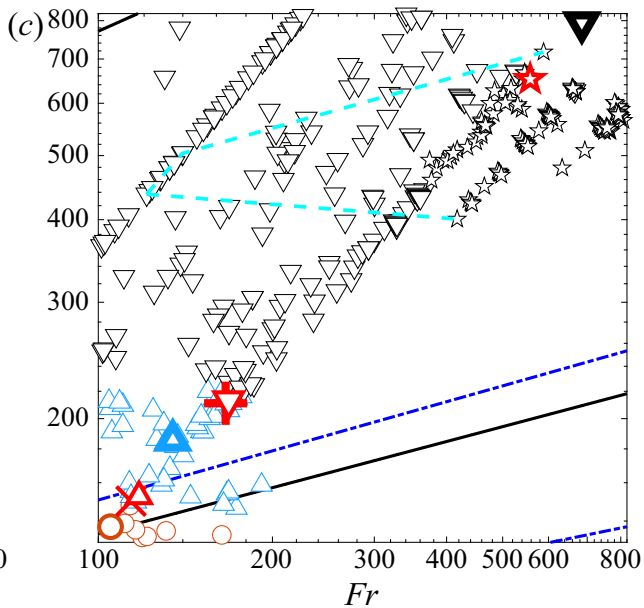

FIgURE 3. (a) Typical dimple shapes for different impact conditions in the multi-dimple regime corresponding to the circled red numbers in $(b)$. Three bamboo-shaped dimples: (1) $D=$ $1.16 \mathrm{~mm}, U=1.7 \mathrm{~m} \mathrm{~s}^{-1}, F r=259$, We $=493$; (2) $D=1.02 \mathrm{~mm}, U=2.1 \mathrm{~m} \mathrm{~s}^{-1}, F r=421$, $W e=617$; (3) $D=0.93 \mathrm{~mm}, U=2.05 \mathrm{~m} \mathrm{~s}^{-1}, F r=463, W e=560$ and a singular telescopic dimple: (4) $D=0.73 \mathrm{~mm}, U=2.38 \mathrm{~m} \mathrm{~s}^{-1}, F r=792, W e=593$. The scale bars are $100 \mu \mathrm{m}$ long. (b) Characterization of the dimples and jets in $\mathrm{Fr}$-We space for drop impacts of immiscible liquids. The two dashed curves are the bounds of the regular bubble-entrapment regime at the bottom of the rebounding crater, measured by Pumphrey \& Elmore (1990) and fitted by Oguz \& Prosperetti (1990). The two solid curves mark the bubble-entrapment region based on our study. This region also includes isolated points of singular jetting, like the one shown in case 4 in panel (a). The symbols correspond to different dimple shapes: $(\bigcirc$, magenta) no pinch-off shallow dimple; $(\triangle$, cyan) dimple pinch-off with bubble going out with the jet; $(\nabla$, black) bubble pinches off and is entrapped inside PP1 drop liquid; (烦, black) telescopic dimple without pinch-off; ( $\square$, blue) drop liquid column breaks up without an air-dimple pinch-off, as shown in figure 4(a); $(\diamond$, green) water entrapped inside PP1 drop liquid, without bubble pinch-off. (c) Enlarged region corresponding to the rectangular box marked by the red dashed lines in $(b)$. The dashed cyan lines (- -, cyan) mark the region where bamboo-like multi-dimples appear (see details in $(a)$ and figure $5 a$ ). The larger symbols with thick edges correspond to conditions similar to those shown in figure 6; $(\bigcirc$, magenta, $\Delta$, cyan and $\nabla$, black) indicate the same dimple shapes as in $(b) ;(\times$, red) first critical pinch-off (first singular jet) at the boundary between no and one bubble pinch-off; $(\triangle$, red) tiny bubble pinched off near first critical pinch off; $(+$, red $)$ secondary critical pinch-off between bubble going out with the jet and bubble entrapped in PP1 drop; ( $\nabla$, red) tiny bubble pinched off near secondary critical pinch-off; (弐, red) singular telescopic dimple. 


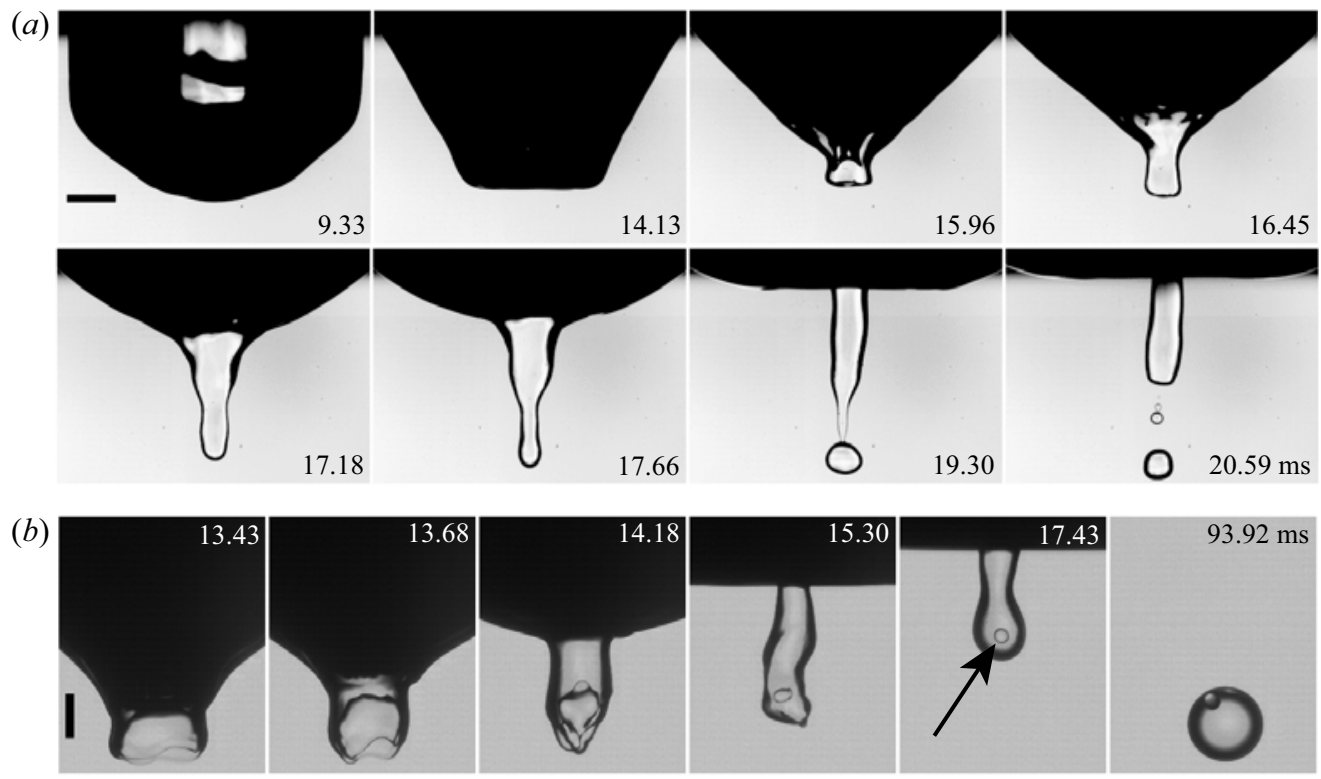

FIGURE 4. Dimple shapes above the upper boundary of the regime of air-dimple formation, above solid line in figure 3(b). (a) Overall view of the pinch-off of a dimple of PP1 drop liquid, without air-bubble pinch-off, for $U=2.29 \mathrm{~m} \mathrm{~s}^{-1}$ and $D=1.63 \mathrm{~mm}$, giving $R e=7862, F r=$ $328, W e=1224$. The drop liquid forms a column which pinches off due to Rayleigh-Plateau instability. There is no air-cavity pinch-off indicated by ( $\square$, blue) in figure $3(b)$. The scale bar is $1 \mathrm{~mm}$. (b) The evolution of water-PP1-water compound drop formation, which corresponds to $\left(\diamond\right.$, green) in figure $3(b) . U=3.57 \mathrm{~m} \mathrm{~s}^{-1}$ and $D=1.20 \mathrm{~mm}$, giving $R e=8902, F r=1099$, $W e=2162$. The black arrow indicates the entrapped water droplet in PP1. In the last frame, the less dense water droplet has risen to the top of the PP1 droplet. The scale bar is $500 \mu \mathrm{m}$. See also supplementary movies 3 and 4 .

\subsection{Capillary waves on dimple}

Figure 3(a) shows a prominent new feature of the dimples, i.e. capillary waves travelling down towards their tips, forming the bamboo-like shapes. Some of these shapes evolve into two or even three pinch-offs, like the double pinch-off shown in figure 5(a). Figure 6 shows the progression of wave shapes along a cut through parameter space, where we keep the drop size fixed at $D=0.94 \pm 0.02 \mathrm{~mm}$, while increasing the impact velocity, to span a range of We from 162 to 1510 . With increasing We, the number of visible wave crests grows from one to three (middle panels) and then the dimple column becomes smooth again (last panels). The second row shows the shapes near the maximum depth, which includes pinch-off shapes and a singular telescopic dimple $(W e=653)$, where no pinch-off occurs, but the fastest jets are ejected out of the crater. This intriguing telescopic shape occurs in a very limited region, within the more common multi-pinch-offs bamboo shapes shown in three of the panels in figure 3(a) and in figure 5(a).

Figure 7 traces the trajectory of the wave troughs along the air-PP1 interface, for four of the cases in figure 6 . We plot the vertical coordinate of the isolated waves versus time, until the maximum depth of the crater and radial collapse. The corresponding movies are listed in figure 6. During the early penetration of the drop into the pool, a prominent trough forms at its outer edge. This prominent wave trough travels down with the crater, producing other troughs ahead of itself. For $W e=301$ (figure $7 a$ ) it emits one wave and for $W e=390$ 
(a)
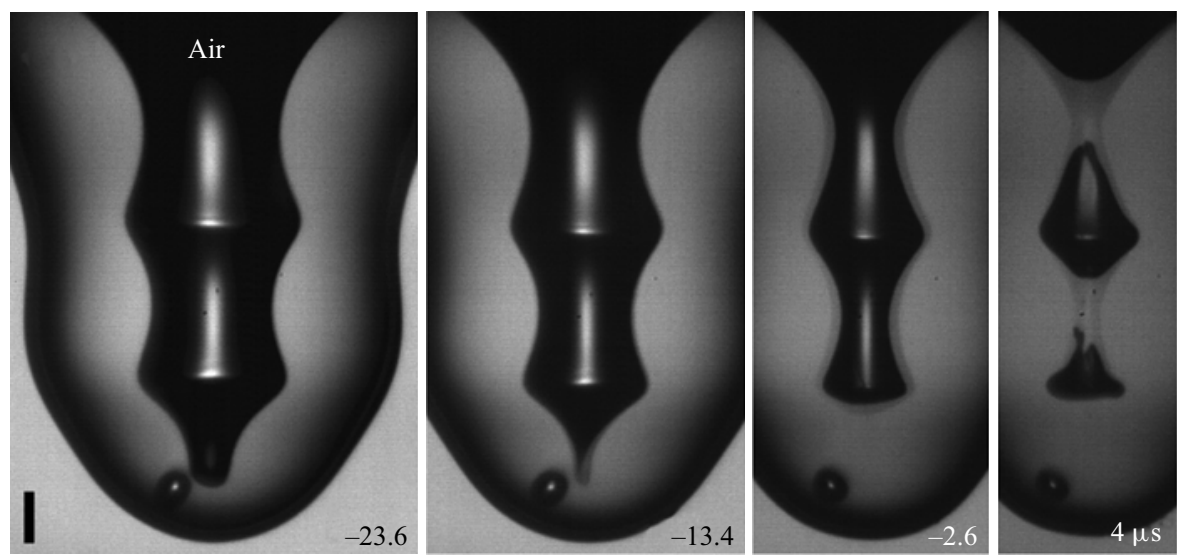

(b)
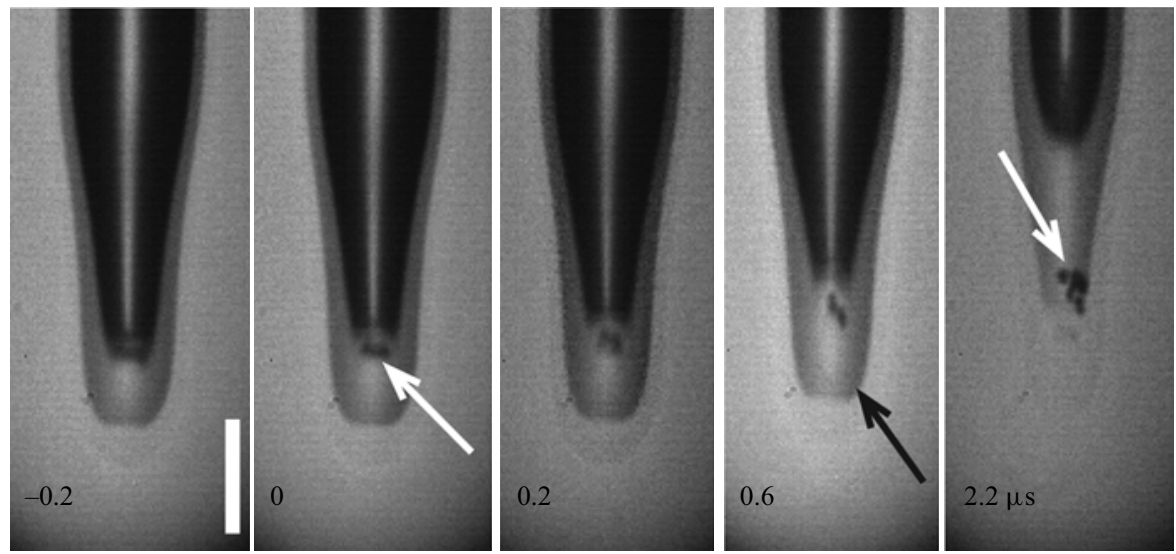

FIGURE 5. (a) Multi-pinch-off bamboo-like dimple shape, corresponding to (2) in figure 3(a). Times are shown relative to the first pinch-off. $(b)$ Micro-bubble shedding from the cusp at the base of the singular jet, for $D=0.82 \mathrm{~mm}, U=2.21 \mathrm{~m} \mathrm{~s}^{-1}, R e=3826, W e=609, \mathrm{Fr}=569$. The white arrows point at the shed micro-bubbles. The image sensor has strong ghosting from every tenth frame (black arrow). The scale bars are $50 \mu \mathrm{m}$ long. See also supplementary movies 5 and 6.

(figure $7 b$ ) it forms two troughs, which reach the bottom before the primary wave. On the other hand, for somewhat larger We these primary waves reach the bottom before the crater reaches maximum depth. A second round of wave troughs are now generated further up the crater wall, which again spawn new waves ahead of them, four in figure 7(c) and three in figure $7(d)$. The growth and shape of the crater makes it difficult to calculate the phase velocity of the troughs. Here, we only show that the vertical speed is in the correct range for capillary waves. In figure $7(b)$ we have fitted the three curves, finding downwards speeds of $0.31,0.32$ and $0.37 \mathrm{~m} \mathrm{~s}^{-1}$. Shorter capillary waves travel faster $u_{c}=\sqrt{2 \pi \sigma /(\rho \lambda)}$, where $\lambda$ is the wavelength. Using the distance between crests in the inset of figure 7(b), we find wavelengths of $0.28,0.19$ and $0.18 \mathrm{~mm}$, predicting quite similar velocities of $0.40,0.49$ and $0.50 \mathrm{~m} \mathrm{~s}^{-1}$. The group velocity for capillary waves is $1.5 \times u_{c}$, supporting the observation of shorter waves emerging ahead of the most prominent wave. Similar wave dynamics is generated by coalescing bubbles, where converging waves lead to partial coalescence (Zhang et al. 2015). 


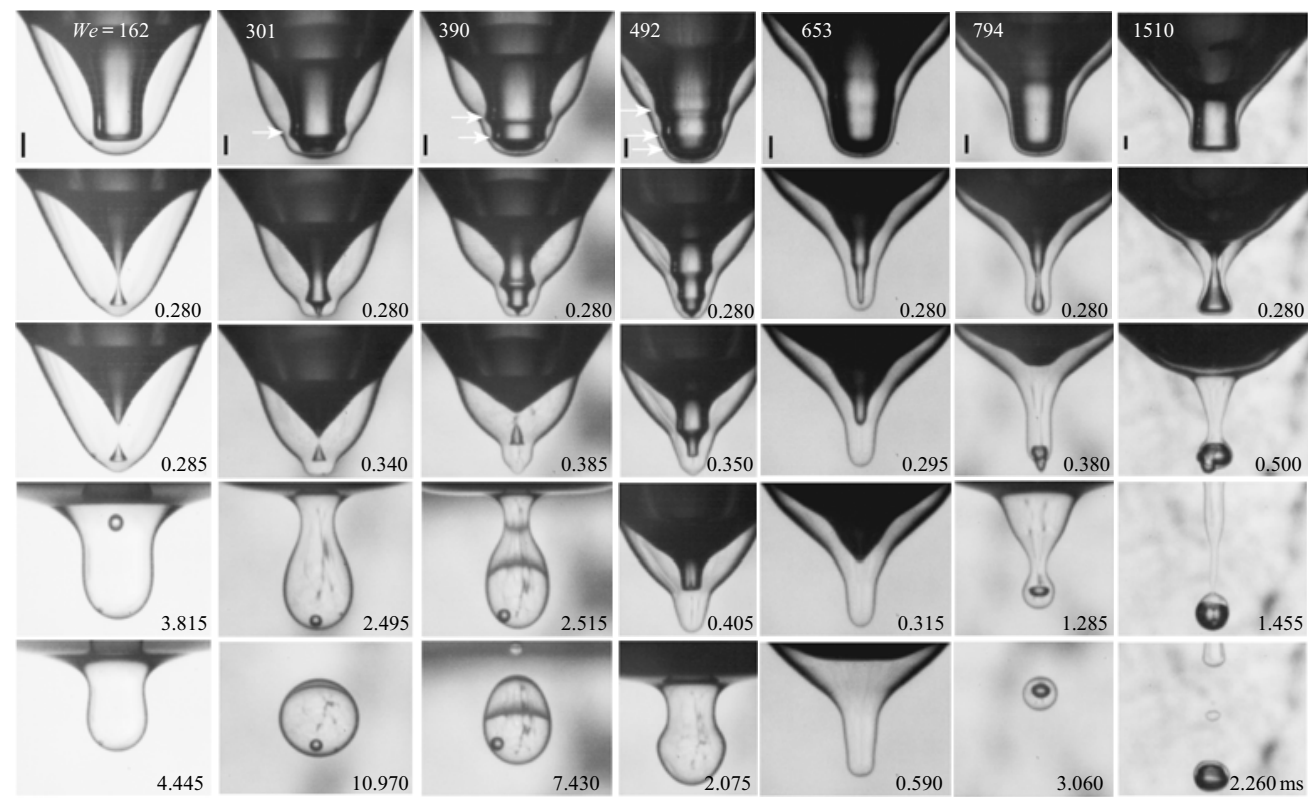

FIGURE 6. Capillary wave shapes on the dimple for a range of $W e$, for similar $D=0.935 \pm$ $0.025 \mathrm{~mm}$ and various impact velocities increasing from left to right: $U=1.09,1.48,1.72,1.91$, $2.23,2.47$ and $3.37 \mathrm{~m} \mathrm{~s}^{-1}$. The arrows point out capillary wave troughs. The scale bars are 200 $\mu \mathrm{m}$ long. See also supplementary movies 7-13.

Figure 8 shows two realizations where the internal jetting is visible inside the air cylinder. From these realizations it becomes clear that the classical picture of singular jets only appearing at the boundaries of the regular bubble-entrapment regime no longer applies and the phase of these capillary waves can induce singular jets at more We values. This is shown for $D=0.92 \mathrm{~mm}$ in figure 9 , with the corresponding jet speeds indicated by the arrow lengths. Here there are three separate We values where no bubble is pinched off and a fast jet is produced (columns 2, 5 and 7). See figure 2 for the shape and break-up of these jets as they emerge from the crater (Yarin 1993; Michon et al. 2017; Lai et al. 2018). The fastest and thinnest jet is observed for the telescopic-dimple case $(W e=653)$, which corresponds to the narrowest angular span of the air cylinder, where the maximum flow volume can be focused into the base of the jet. This narrow shape looks reminiscent of the capillary-driven retraction of a conical drop, studied by Brasz, Berny \& Bird (2018b).

\subsection{Cross-over}

The above dimple shapes show that not only can the boundary conditions break the axisymmetry of the collapse of a pinching air cylinder (Burton et al. 2005; Keim et al. 2006; Enriquez et al. 2012; Lai 2012), but they can also imprint a large variety of axial shapes on the free surface of the dimple, thereby modifying its singular collapse.

What is the role of capillary waves in setting up the dimple for the inertial focusing? For the singular jets the dimple dynamics has until recently been formulated in the self-similar capillary-inertial formalism (Zeff et al. 2000; Duchemin et al. 2002; Deike et al. 2018), while the final cylindrical collapse has most recently been shown to follow pure inertial focusing (Thoroddsen et al. 2018; Gordillo \& Rodríguez-Rodríguez 2019). One can therefore expect a dynamical transition in the vicinity of the final jet formation. 

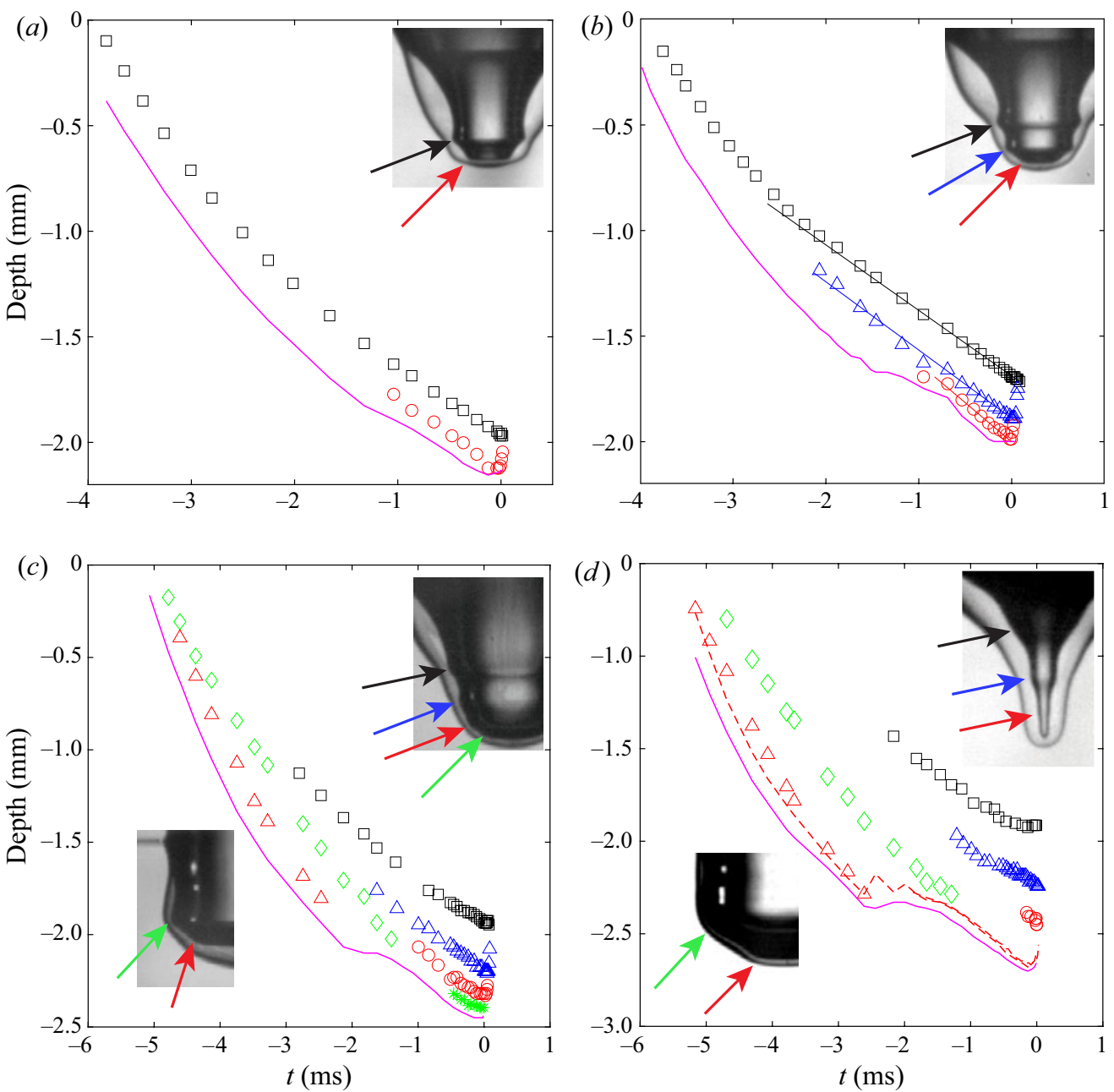

FIgURE 7. Trajectories of the wave troughs along the crater free surface for four cases from figure 6: $(a) W e=301 ;(b) W e=390 ;(c) W e=492 ;(d) W e=653$. The magenta continuous curves mark the bottom penetration of the PP1 droplet at the centreline. The dashed red curve in $(d)$ marks the bottom of the air crater. The coloured arrows point out the troughs tracked by the corresponding coloured symbols. The original red and green tracks in $(c, d)$ reach the bottom of the crater, while other waves are generated further up. The slopes of the lines in $(b)$ are -0.31 , -0.32 and $-0.37 \mathrm{~m} \mathrm{~s}^{-1}$ starting from the top curve.

In figure 10(a) we track the radius of the pinch-off neck for a pinch-off case of the bamboo-shaped dimple, shown in the inset. There is here a clear cross-over in the nature of the dynamics from capillary-inertial $R \sim t^{2 / 3}$ to purely inertial with $R \sim t^{0.55}$ at $t_{c} \simeq 65 \mu \mathrm{s}$ before pinch-off, as marked by the arrow. Figure $10(b)$ shows that the cross-over time scales with the impact time $t_{c} \simeq 0.235 R_{d} / U$ for the cases where a small bubble is pinched off. On the other hand, for the fastest singular jetting the cross-over time occurs much earlier, irrespective of We. The vertical arrows indicate a lower bound for $t_{c}$, as the data have reached the end of the 180 frame video clip without deviating from the $\simeq 0.55$ power-law exponent. This difference is explicitly shown in the log-log plots in figure $10(c, d)$ where the singular collapse in $(d)$ follows the inertial power law 
(a)

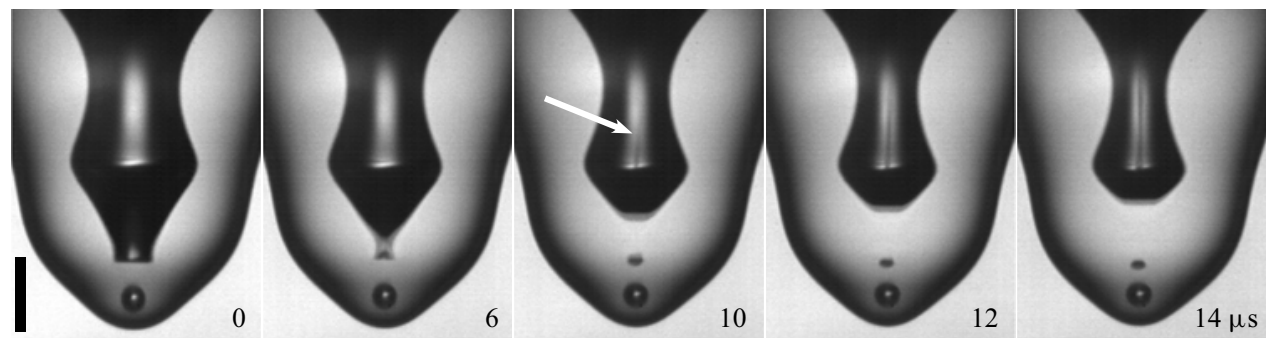

(b)

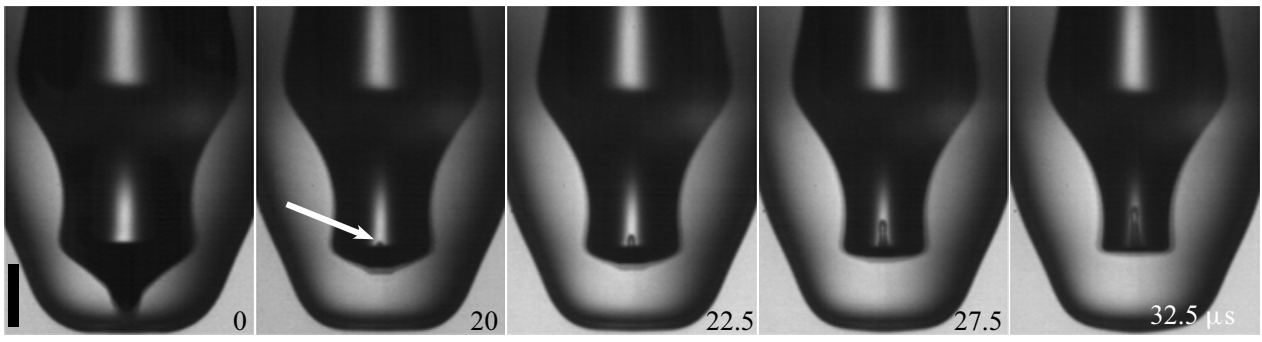

FIGURE 8. Early-time jet visible inside the air dimple. $(a, b)$ Present the different singular jets in the cavity visualized by our imaging method. The white arrows indicate jettings inside the cavity. The scale bars are $100 \mu \mathrm{m}$ long. See also supplementary movies 14 and 15 .

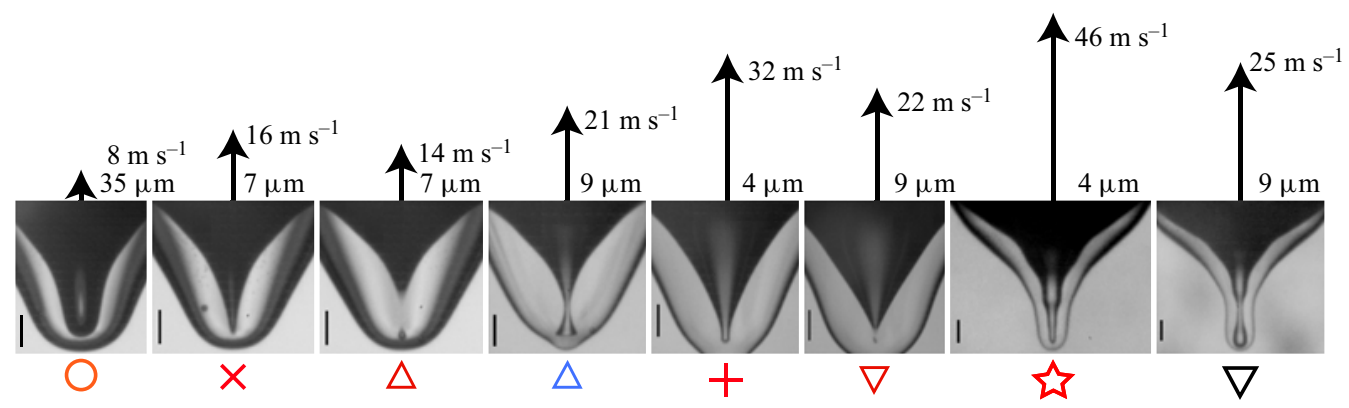

FigURE 9. Overview of dimple shape and jet velocity versus $W e$, for drop size $0.92 \mathrm{~mm}$. The arrow lengths indicate the jet velocities. The Weber number grows from left to right $(W e=$ $137,139,153,186,211,213,653,794)$. The scale bars are $200 \mu \mathrm{m}$. See also supplementary movies 7, 11 and 12 .

for at least $500 \mu \mathrm{s}$. We note that the measured exponent $\simeq 0.55$, being significantly larger than the inertial power law of 0.5 , is consistent with previous measurements of bubble pinch-off (Bergmann et al. 2006; Thoroddsen et al. 2007a), which is explained by the slow asymptotic observed in the corresponding theory (Eggers et al. 2007; Gordillo \& Fontelos 2007).

This cross-over from capillary-inertial scaling can also be revealed by the local prefactor in the capillary-inertial power law, i.e. $C=R /\left(\sigma t^{2} / \rho\right)^{1 / 3}$ (Burton, Rutledge \& Taborek 2004, 2007; Deblais et al. 2018). Figure 11 shows that $C$ is approximately constant during the early capillary-driven stage, but then increases steadily on approaching the final pinch-off. The value of $C$ is linearly proportional to the radial velocity $\mathrm{d} R / \mathrm{d} t=$ $(2 C / 3)(\sigma / \rho)^{1 / 3} t^{-1 / 3}$ and the rise in $C$ indicates the accelerated collapse due to the inertial focusing, exceeding the speed of the capillary-driven motions. The value of $C=3.7$ is much larger than for a drop pinching off from a nozzle, which has recently been 

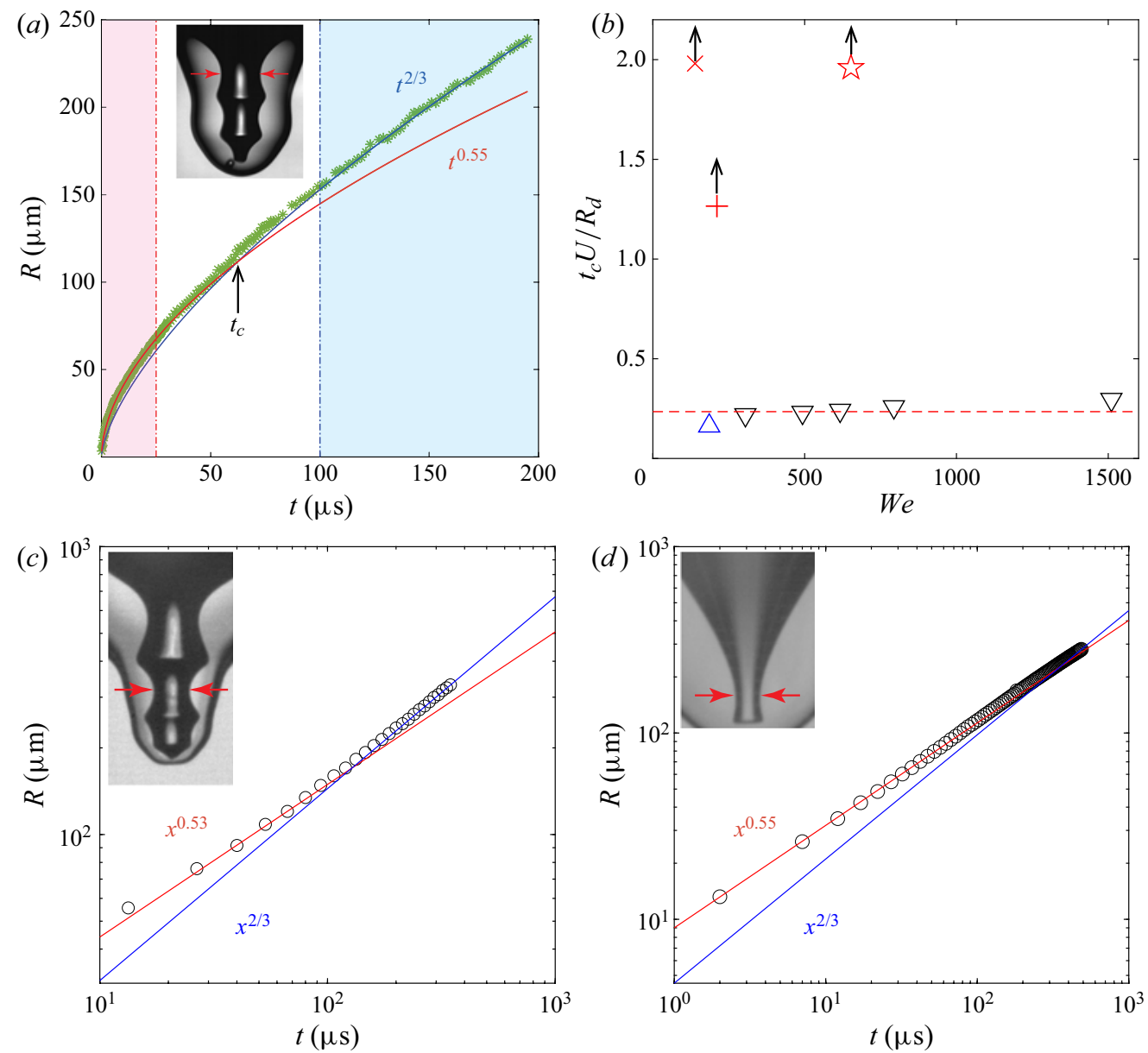

FIgURE 10. (a) Scaling of the dimple radius vs time before pinch-off, for $U=2.05 \mathrm{~m} \mathrm{~s}^{-1}$, $D=1.02 \mathrm{~mm}$ and $R e=4418, F r=421, W e=617$. There is a transition of power-law exponents from $2 / 3$ to 0.55 closest to the pinch-off. The background shading marks the validity of each, with the arrow indicating the approximate cross-over time $t_{c}$. The data are taken from two movie clips spanning time scales from $100 \mathrm{~ns}$ to $200 \mu \mathrm{s}$ before pinch-off. The corresponding log-log-plots are included in the supplementary material. $(b)$ The cross-over time $t_{c}$ normalized by the impact time $D / U$ vs $W e$, for dimple pinch-off $(\triangle$, cyan and $\nabla$, black) and singular jets $(\times$, red, + , red

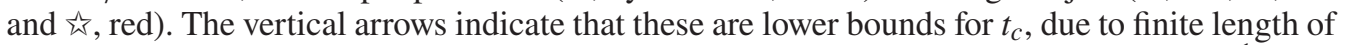
the movie clips. $(c, d)$ Show the pinch-off under different conditions. (c) $U=1.72 \mathrm{~m} \mathrm{~s}^{-1}, D=$ $1.2 \mathrm{~mm}$ and $R e=4215, F r=259, W e=493$. (d) Singular jetting without bubble pinch-off, for $U=1.21 \mathrm{~m} \mathrm{~s}^{-1}, D=0.99 \mathrm{~mm}, \operatorname{Re}=2531, F r=152$, We $=209$. The horizontal red arrows indicate the location where the minimum dimple radius is tracked.

investigated by Deblais et al. (2018), where $C \leq 0.717$. If we use the density of the water pool and the interface tension between drop and pool, $C_{\text {water }}$ reduces to 1.95 . This indicates that the inertia of the pool is more important, during the early motions, than that of the thin drop layer. We also find that the value of $C$ is fairly constant over the range of We, as shown in figure $11(d)$. Larger We will increase the crater size and thereby reduce the thickness of the drop liquid surrounding the dimple, as is clearly seen in figures 6 and 9 . 

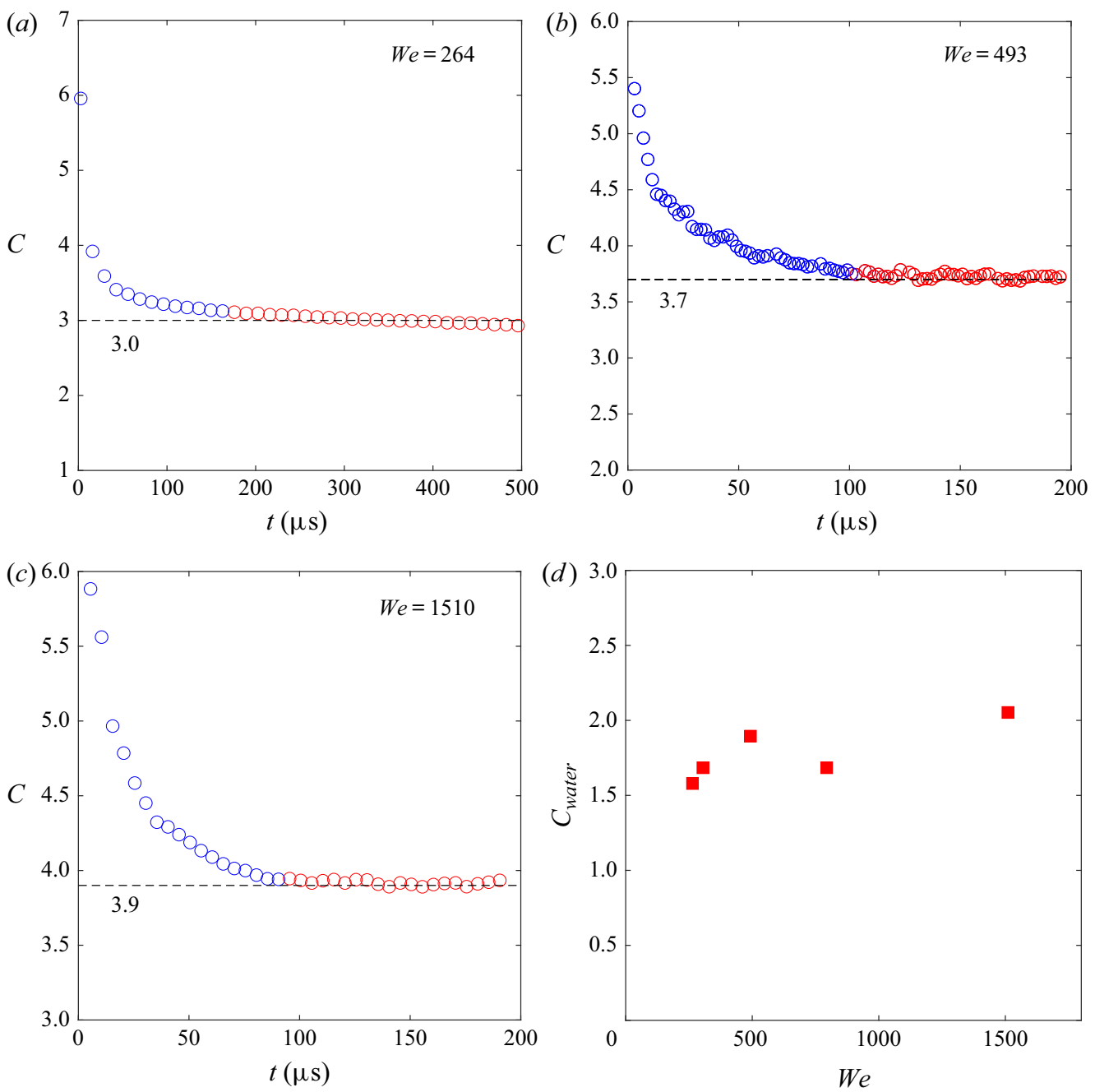

FIGURE 11. The instantaneous prefactor $C$ of the capillary-inertial scaling, as a function of time before the pinch-off collapse, for different Weber numbers: $(a) W e=264,(b) W e=493$, (c) $W e=1510$. Values are calculated with PP1 properties. The approach to the singularity goes from right to left $(t \rightarrow 0)$. The initial dynamics follows the capillary-inertial power law with a constant prefactor (red circles), while closer to the singularity the velocity speeds up (blue circles), indicating inertial acceleration and power-law scaling transition from $2 / 3$ to 0.55 . (d) Prefactors calculated with water density and water-PP1 interfacial tension, which is indicated by the subscript $C_{\text {water }}=0.53 C$.

The prefactor during the final inertial collapse arises from the balance of two inertial terms, acceleration and convection (Eggers et al. 2007). In the final stage the prefactor should therefore not depend on the surface tension or the hydrostatic pressure. We are therefore led to the simple scaling of

$$
R(t)=C_{\text {inertia }} \sqrt{D U} t^{1 / 2},
$$

which gives a fairly constant value of $C_{\text {inertia }} \simeq 0.30 \pm 0.04$, over the range of We numbers tested, as shown in figure 12. This value is consistent with data from 

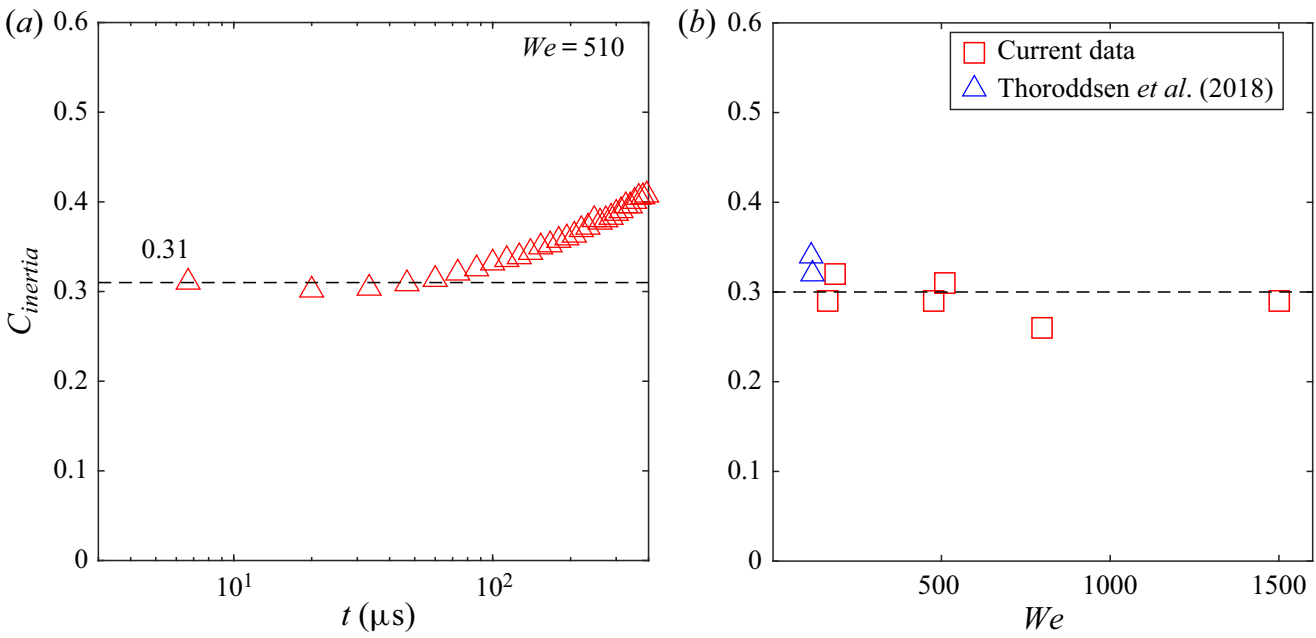

FIGURE 12. Scaling prefactor during the final inertial collapse, using (3.1). (a) The asymptotic value of the prefactor $C_{\text {inertia }}$ vs time before pinch-off, for $W e=510$. $(b)$ The coefficient over a range of We, including data from Thoroddsen et al. (2018) (their figure 5), where the drop size, density and surface tension are quite different from the current study.

Thoroddsen et al. (2018), included in the figure. Keep in mind that these data are for larger drops and different liquid properties. However, the viscosity in that study is larger and more systematic testing is needed to verify this relation, over a wider range of properties. Relating this formula to the bursting of a static bubble, one can use the capillary-inertial velocity, based on the bubble radius $R_{b}, u_{\sigma}=\sqrt{\sigma /\left(\rho R_{b}\right)}$, to get $R(t) \sim\left(\sigma R_{b} / \rho\right)^{1 / 4} t^{1 / 2}$, in accordance with Burton et al. (2005).

\section{Discussion and conclusions}

Herein, we report a plethora of new dimple shapes, which occur following a drop impact on an immiscible pool. This includes multiple pinch-off bamboo shapes from capillary waves, which mould the axial shape of the dimple before its final purely inertial collapse. This destroys any hope of finding a universal self-similar dimple shape. We have also identified many discrete We-values where singular jetting is observed.

Questions remain: what determines the minimum diameter of the singular dimple and thereby its maximum jetting velocity? The smallest singular dimple width is here $\simeq 12 \mu \mathrm{m}$ which is similar to the $15 \mu \mathrm{m}$ observed by Thoroddsen et al. (2018), who used a liquid which is an order of magnitude more viscous. This suggests that a viscous cutoff is not at play for the much lower viscosity of our PP1 drop (Castrejón-Pita et al. 2015; Brasz et al. 2018a). We can speculate that cavitation or vortex-shedding instability (Thoraval et al. 2012) in the cusp at the base of the jet prevents smaller jet sizes, as we see by the micro-bubbles shed at the base of the jet in figure 5(b). The expansion of the micro-bubble volume in the last panel indicates the large localized pressure driving up the singular jet (Tran et al. 2016; Thoroddsen et al. 2018; Gordillo \& Rodríguez-Rodríguez 2019).

We conclude that our singular jets differ from bubble-bursting jets, in fundamental ways. First, the dimple shapes are not self-similar during the final collapse (Duchemin et al. 2002; Lai et al. 2018). Secondly, figures 6 and 9 show clearly that the Ohnesorge number, which is approximately constant, $O h=\mu_{d} / \sqrt{\rho_{d} R_{c} \sigma_{d}} \simeq 0.0054$, based on the 
maximum crater radius $R_{c}=1.1 \mathrm{~mm}$, is not sufficient to describe the varied dynamics, as is suggested for bursting-bubble jetting (Gañán-Calvo 2017, 2018; Gordillo \& Rodríguez-Rodríguez 2019). It is a clear indication of the extreme focusing of energy that the maximum jetting velocity $v_{j}=46 \mathrm{~m} \mathrm{~s}^{-1}$ is $\sim 580 \pm 30$ times the capillary velocity $v_{\sigma}=\sqrt{\sigma_{d} /\left(\rho_{d} R_{c}\right)}$. This is an order of magnitude faster than predicted for the bursting bubbles (Deike et al. 2018; Gañán-Calvo 2018; Gordillo \& Rodríguez-Rodríguez 2019). The jet diameters of $4 \mu \mathrm{m}$ are also two orders of magnitude thinner than those predicted by the bursting-bubble theory (Gañán-Calvo 2018), see also the Comment of Gordillo \& Rodríguez-Rodríguez (2018), who question the underlying scaling of this theory. One clear difference from bursting bubbles is the residual velocity field, which exists at the start of crater collapse. Another is the generation of capillary waves at the edge of the impacting drop.

The cross-over from capillary-driven motions to inertial focusing is not unique to immiscible impacts. The inertial focusing in Thoroddsen et al. (2018) also appears to be preceded by a capillary-inertial stage, but is not mentioned in that work. We include one similar example in the supplementary material.

Finally, we point out that while the final inertial focusing occurs on tens of $\mu \mathrm{m}$ length scales, the larger-scale liquid inertia is here a function of time, owing to the local thickness of the drop liquid around the dimple, which also becomes thinner with increasing We. This effect can be investigated by changing the relative density of the two liquids, in future experiments.

\section{Acknowledgements}

This study was supported by King Abdullah University of Science and Technology (KAUST) under grant URF/1/3727-01-01. We acknowledge useful discussions with J. Eggers, C. Y. Lei and L. Deike.

\section{Declaration of interests}

The authors report no conflict of interest.

\section{Supplementary movies and material}

Supplementary movies and material are available at https://doi.org/10.1017/jfm.2020. 694.

\section{REFERENCES}

Antkowiak, A., Bremond, N., Le Dizès, S. \& Villermaux, E. 2007 Short-term dynamics of a density interface following an impact. J. Fluid Mech. 577, 241-250.

Bartolo, D., Josserand, C. \& Bonn, D. 2006 Singular jets and bubbles in drop impact. Phys. Rev. Lett. 96 (12), 124501.

Bergmann, R., van der Meer, D., Stijnman, M., Sandtke, M., Prosperetti, A. \& Lohse, D. 2006 Giant bubble pinch-off. Phys. Rev. Lett. 96 (15), 154505.

Brasz, C. F., Bartlett, C. T., Walls, P. L. L., Flynn, E. G., Yu, Y. E. \& Bird, J. C. $2018 a$ Minimum size for the top jet drop from a bursting bubble. Phys. Rev. Fluids 3 (7), 074001.

Brasz, C. F., BERnY, A. \& BIRD, J. C. $2018 b$ Threshold for discretely self-similar satellite drop formation from a retracting liquid cone. Phys. Rev. Fluids 3 (10), 104002.

Brenner, M. P., Eggers, J., Joseph, K., Nagel, S. R. \& Shi, X. 1997 Breakdown of scaling in droplet fission at high Reynolds number. Phys. Fluids 9 (6), 1573-1590. 
Burton, J. C., Rutledge, J. E. \& TAborek, P. 2004 Fluid pinch-off dynamics at nanometer length scales. Phys. Rev. Lett. 92 (24), 244505.

Burton, J. C., Rutledge, J. E. \& TABorek, P. 2007 Fluid pinch-off in superfluid and normal He 4. Phys. Rev. E 75 (3), 036311.

Burton, J. C., WALdRep, R. \& TABorek, P. 2005 Scaling and instabilities in bubble pinch-off. Phys. Rev. Lett. 94 (18), 184502.

Castrejón-Pita, J. R., Castrejón-Pita, A. A., Thete, S. S., Sambath, K., Hutchings, I. M., Hinch, J., LISTER, J. R. \& BASARAN, O. A. 2015 Plethora of transitions during breakup of liquid filaments. Proc. Natl Acad. Sci. 112 (15), 4582-4587.

Chandrasekhar, S. 1992 The Mathematical Theory of Black Holes. Oxford University Press.

CHOPTUIK, M. W. 1993 Universality and scaling in gravitational collapse of a massless scalar field. Phys. Rev. Lett. 70 (1), 9-12.

DAS, S. P. \& Hopfinger, E. J. 2008 Parametrically forced gravity waves in a circular cylinder and finite-time singularity. J. Fluid Mech. 599, 205-228.

DAY, R. F., Hinch, E. J. \& Lister, J. R. 1998 Self-similar capillary pinchoff of an inviscid fluid. Phys. Rev. Lett. 80 (4), 704-707.

Deblais, A., Herrada, M. A., Hauner, I., Velikov, K. P., Van Roon, T., Kellay, H., Eggers, J. \& Bonn, D. 2018 Viscous effects on inertial drop formation. Phys. Rev. Lett. 121 (25), 254501.

Deike, L., Ghabache, E., Liger-Belair, G., Das, A. K., Zaleski, S., Popinet, S. \& Séon, T. 2018 Dynamics of jets produced by bursting bubbles. Phys. Rev. Fluids 3 (1), 013603.

Duchemin, L., Popinet, S., Josserand, C. \& Zaleski, S. 2002 Jet formation in bubbles bursting at a free surface. Phys. Fluids 14 (9), 3000-3008.

EgGers, J. 1997 Nonlinear dynamics and breakup of free-surface flows. Rev. Mod. Phys. 69 (3), 865-929.

Eggers, J. \& Fontelos, M. A. 2015 Singularities: Formation, Structure and Propagation. Cambridge University Press.

Eggers, J., Fontelos, M. A., Leppinen, D. \& Snoeijer, J. H. 2007 Theory of the collapsing axisymmetric cavity. Phys. Rev. Lett. 98 (9), 094502.

Enriquez, O. R., Peters, I. R., Gekle, S., Schmidt, L. E., Lohse, D. \& VAn der Meer, D. 2012 Collapse and pinch-off of a non-axisymmetric impact-created air cavity in water. J. Fluid Mech. 701, 40-58.

Fujimatsu, T., Fujita, H., Hirota, M. \& OKada, O. 2003 Interfacial deformation between an impacting water drop and a silicone-oil surface. J. Colloid Interface Sci. 264 (1), 212-220.

GAÑÁN-CALVO, A. M. 2017 Revision of bubble bursting: universal scaling laws of top jet drop size and speed. Phys. Rev. Lett. 119 (20), 204502.

Gañán-CALVo, A. M. 2018 Scaling laws of top jet drop size and speed from bubble bursting including gravity and inviscid limit. Phys. Rev. Fluids 3 (9), 091601.

GeKle, S., Gordillo, J. M., VAn Der Meer, D. \& Lohse, D. 2009 High-speed jet formation after solid object impact. Phys. Rev. Lett. 102 (3), 034502.

Gordillo, J. M. \& Fontelos, M. A. 2007 Satellites in the inviscid breakup of bubbles. Phys. Rev. Lett. 98 (14), 144503.

Gordillo, J. M. \& Rodríguez-Rodríguez, J. 2018 Comment on "Revision of bubble bursting: universal scaling laws of top jet drop size and speed". Phys. Rev. Lett. 121, 269401.

Gordillo, J. M. \& RodríGUEZ-RodríGueZ, J. 2019 Capillary waves control the ejection of bubble bursting jets. J. Fluid Mech. 867, 556-571.

Jain, U., JALAAL, M., LOHSE, D. \& VAN DER MEER, D. 2019 Deep pool water-impacts of viscous oil droplets. Soft Matter 15 (23), 4629-4638.

Jian, Z., Channa, M. A., Kherbeche, A., Chizari, H., Thoroddsen, S. T. \& Thoraval, M.-J. 2020 To split or not to split: dynamics of an air disk formed under a drop impacting on a pool. Phys. Rev. Lett. 124, 184501.

Keim, N. C., Møller, P., Zhang, W. W. \& Nagel, S. R. 2006 Breakup of air bubbles in water: memory and breakdown of cylindrical symmetry. Phys. Rev. Lett. 97 (14), 144503.

LAI, L. 2012 Curvature singularity in the asymmetric breakup of an underwater air bubble. Phys. Fluids 24 (10), 102106.

Lai, C. Y., Eggers, J. \& Deike, L. 2018 Bubble bursting: universal cavity and jet profiles. Phys. Rev. Lett. 121 (14), 144501. 
Lhuissier, H., Sun, C., Prosperetti, A. \& Lohse, D. 2013 Drop fragmentation at impact onto a bath of an immiscible liquid. Phys. Rev. Lett. 110 (26), 264503.

Liow, J. L. 2001 Splash formation by spherical drops. J. Fluid Mech. 427, 73-105.

LIOW, J. L. \& COLE, D. E. 2007 Bubble entrapment mechanisms during the impact of a water drop. In 16th Australasian Fluid Mechanics Conference (AFMC), School of Engineering, the University of Brisbane, Australia, pp. 866-869.

Longuet-Higgins, M. S. 1983 Bubbles, breaking waves and hyperbolic jets at a free surface. J. Fluid Mech. 127, 103-121.

Michon, G.-J., Josserand, C. \& SÉon, T. 2017 Jet dynamics post drop impact on a deep pool. Phys. Rev. Fluids 2 (2), 023601.

Murphy, D. W., Li, C., D’Albignac, V., Morra, D. \& Katz, J. 2015 Splash behaviour and oily marine aerosol production by raindrops impacting oil slicks. J. Fluid Mech. 780, 536-577.

Oguz, H. N. \& Prosperetti, A. 1990 Bubble entrainment by the impact of drops on liquid surfaces. J. Fluid Mech. 219, 143-179.

Prosperetti, A. \& Oguz, H. N. 1993 The impact of drops on liquid surfaces and the underwater noise of rain. Annu. Rev. Fluid Mech. 25 (1), 577-602.

Pumphrey, H. C. \& Elmore, P. A. 1990 The entrainment of bubbles by drop impacts. J. Fluid Mech. 220, 539-567.

REIN, M. 1996 The transitional regime between coalescing and splashing drops. J. Fluid Mech. 306, $145-165$.

Schmidt, L. E., Keim, N. C., Zhang, W. W. \& NAgEL, S. R. 2009 Memory-encoding vibrations in a disconnecting air bubble. Nat. Phys. 5 (5), 343-346.

Tagawa, Y., Oudalov, N., Visser, C. W., Peters, I. R., van der Meer, D., Sun, C., Prosperetti, A. \& Lohse, D. 2012 Highly focused supersonic microjets. Phys. Rev. X 2 (3), 031002.

Terwagne, D., Mack, N., Dorbolo, S., Gilet, T., Raty, J. Y. \& Vandewalle, N. 2009 The mayonnaise droplet. Chaos 19 (4), 41105.

Thoraval, M.-J., LI, Y. \& Thoroddsen, S. T. 2016 Vortex-ring-induced large bubble entrainment during drop impact. Phys. Rev. E 93 (3), 033128.

Thoraval, M.-J., Takehara, K., Etoh, T. G., Popinet, S., Ray, P., Josserand, C., Zaleski, S. \& Thoroddsen, S. T. 2012 von Kármán vortex street within an impacting drop. Phys. Rev. Lett. 108 (26), 264506.

Thoroddsen, S. T., Etoh, T. G. \& Takehara, K. $2007 a$ Experiments on bubble pinch-off. Phys. Fluids 19 (4), 042101.

Thoroddsen, S. T., Etoh, T. G. \& TAKehara, K. $2007 b$ Microjetting from wave focusing on oscillating drops. Phys. Fluids 19 (5), 052101.

Thoroddsen, S. T., Takehara, K., Etoh, T. G. \& Ohl, C. D. 2009 Spray and microjets produced by focusing a laser pulse into a hemispherical drop. Phys. Fluids 21 (11), 112101.

Thoroddsen, S. T., Takehara, K., Nguyen, H. D. \& Etoh, T. G. 2018 Singular jets during the collapse of drop-impact craters. J. Fluid Mech. 848, R3.

Tran, T. T., Lee, E. G., Lee, I. S., Woo, N. S., Han, S. M., Kim, Y. Ju \& Hwang, W. R. 2016 Hydrodynamic extensional stress during the bubble bursting process for bioreactor system design. Korea-Aust. Rheol. J. 28 (4), 315-326.

WALls, P. L., HENAUX, L. \& BIRD, J. C. 2015 Jet drops from bursting bubbles: how gravity and viscosity couple to inhibit droplet production. Phys. Rev. E 92 (2), 021002.

YARIN, A. L. 1993 Free Liquid Jets and Films: Hydrodynamics and Rheology. Longman Publishing Group.

ZefF, B. W., Kleber, B., Fineberg, J. \& Lathrop, D. P. 2000 Singularity dynamics in curvature collapse and jet eruption on a fluid surface. Nature 403 (6768), 401-404.

Zhang, F., Thoraval, M.-J., Thoroddsen, S. T. \& TABorek, P. 2015 Partial coalescence from bubbles to drops. J. Fluid Mech. 782, 209-239. 